\title{
Concordancia diagnóstica de capsulitis adhesiva por resonancia magnética entre centros de imagenología y una clínica de reconstrucción articular con correlación quirúrgica
}

\author{
Diagnostic concordance of Magnetic Resonance Imaging of Adhesive Capsulitis, \\ between Imaging Centers and a Shoulder Clinic, with surgical correlation
}

\author{
Santa-María-Gasca NE, ${ }^{*}$ Aguirre-Rodríguez VH, ${ }^{\ddagger}$ Valdés-Montor JF, $*$ \\ Mejía-Terrazas GE, ${ }^{\S}$ Valero-González FS"
}

Hospital Ángeles Pedregal.

RESUMEN. Introducción: Los hallazgos en resonancia magnética (IRM) presentan una adecuada sensibilidad y especificidad para el diagnóstico de capsulitis adhesiva; sin embargo, existe una baja correlación diagnóstica entre la interpretación realizada en los centros de imagenología y la de la clínica de reconstrucción articular y los hallazgos quirúrgicos. Objetivo: Conocer la correlación del diagnóstico de capsulitis adhesiva por IRM entre centros de imagenología y la clínica de reconstrucción articular con posterior comprobación quirúrgica. Material y métodos: Estudio retrospectivo, observacional y comparativo en un grupo de 41 pacientes con diagnóstico de capsulitis adhesiva, comparando la concordancia de los reportes de IRM de distintos centros de imagenología y la clínica de reconstrucción articular, se corroboró quirúrgicamente en 10 casos. Se determinó el índice $\kappa$ entre ambas interpretaciones y los hallazgos quirúrgicos. Resultados: La concordancia de los reportes analizados por el cirujano de hombro de la clínica de reconstrucción articular y los reportes de los centros de imagenología fue baja con un índice $\kappa$ de 0.12 , en todos los casos quirúrgicos se corroboró la presencia de capsulitis ad-
ABSTRACT. Introduction: Despite the MRI findings present an adequate sensitivity and specificity for the diagnosis of adhesive capsulitis, there is a poor correlation between the imaging results in imaging centers and the diagnosis of a shoulder surgeon and the surgical findings. Objective: To evaluate the correlation of the diagnosis of adhesive capsulitis by MRI between imaging centers and the shoulder and elbow surgery service, with subsequent surgical verification. Material and methods: Retrospective, observational and comparative study in 41 patients with adhesive capsulitis diagnosis, the concordance among the MRI reports of different radiology cabinets and the Joint reconstruction clinic was calculated, in 10 cases the diagnosis was corroborated by surgery. The $\kappa$ index was determinated between both interpretations and the surgical findings. Results: The concordance among the reports analyzed by the shoulder surgeon of the Joint reconstruction clinic and the cabinets was low, with a $\kappa$ index of 0.12 , in all the surgical cases the presence of adhesive capsulitis was proved with a $\kappa$ index of 0.10 with respect to the cabinets reports. Conclusion: The imaging findings described

\section{Nivel de evidencia: IV}

* Cirujano Ortopedista. Alumno del Curso de Posgrado de Alta Especialidad en Reconstrucción Articular de Hombro y Codo. Facultad Mexicana de Medicina, Universidad La Salle en el Hospital Ángeles Pedregal. Ciudad de México.

‡ Cirujano Ortopedista, Clínica de Reconstrucción Articular, Hospital Ángeles Pedregal. Profesor adjunto del Curso de Postgrado de Alta Especialidad en Reconstrucción Articular de Hombro y Codo. Facultad Mexicana de Medicina, Universidad La Salle en el Hospital Ángeles Pedregal. Ciudad de México.

${ }^{\S}$ Médico Anestesiólogo-Algólogo. Maestro en ciencias médicas. Clínica del Dolor en el Hospital Ángeles México. Ciudad de México.

" Cirujano Ortopedista, Cirugía de Hombro y Codo. Clínica de Reconstrucción Articular, Hospital Ángeles Pedregal. Profesor Titular del Curso de Postgrado de Alta Especialidad en Reconstrucción Articular de Hombro y Codo. Facultad Mexicana de Medicina, Universidad La Salle en el Hospital Ángeles Pedregal. Ciudad de México.

Dirección para correspondencia:

Dr. Fernando Sergio Valero González

Clínica de Reconstrucción Articular. Hospital Ángeles Pedregal

Periférico Sur Núm. 3707-970, Col. Héroes de Padierna, Alcaldía Magdalena Contreras, CP. 10700, CDMX, Tel: (55) 5135-1417

E-mail: shoulder.elbow@gmail.com

Este artículo puede ser consultado en versión completa en: www.medigraphic.com/actaortopedica 
hesiva, el índice $\kappa$ con los reportes de los centros de imagenología fue de 0.10. Conclusión: Los hallazgos en la IRM descritos en la literatura son confiables para el diagnóstico capsulitis adhesiva. La baja concordancia con los centros de imagenología nos orienta a pensar que es una entidad subdiagnosticada por esas instituciones.

Palabras clave: Diagnóstico, resonancia magnética, capsulitis, hombro, cirugía.

\section{Introducción}

La capsulitis adhesiva es un padecimiento descrito por Simon Emmanuel Duplay como periartritis escapulohumeral, en 1943 Codman acuña el término de hombro congelado, quien lo describió como un síndrome difícil de definir, difícil de tratar y difícil de explicar. ${ }^{1,2}$ Es Neviaser en 1945 quien lo redefine como capsulitis adhesiva, ${ }^{1,3}$ se le conoce también como artrofibrosis. ${ }^{1}$ Su curso clínico se acompaña de dolor localizado que se exacerba al movimiento, de predominio nocturno, ${ }^{4}$ rigidez y disfunción.

Actualmente su prevalencia real se desconoce, pero se estima que se presenta en $5.3 \%$ de la población ${ }^{1}$ en el rango de 40 a 65 años de edad, ${ }^{5}$ afecta al género femenino en $70 \%$ de los $\operatorname{casos}^{1}$ y hasta en $20 \%$ de la población con diabetes, en particular en la extremidad no dominante se manifiesta entre 40 y $50 \%$ de los casos de forma bilateral, ${ }^{1}$ condición que afecta sobre todo a la población diabética y a los que muestran el primer cuadro antes de los 50 años. ${ }^{5}$ Es una patología autolimitada, con una duración entre 1 y 3.5 años, con un promedio de 30.5 meses. El diagnóstico diferencial debe realizarse con lesiones del manguito de los rotadores, tendinitis o bursitis calcificada o con un proceso degenerativo glenohumeral. ${ }^{4}$ Según su etiología se dividen en primarias cuando es idiopática y en secundarias cuando se asocian a desgarros de manguito rotador o traumatismos. ${ }^{1,6,7}$ Los factores de riesgo asociados al desarrollo de capsulitis adhesiva son el género femenino, edad mayor de 40 años, historia familiar de capsulitis adhesiva, inmovilización prolongada de hombro, hipercolesterolemia, enfermedades autoinmunes, presencia del antígeno HLA B27 positivo, diabetes mellitus tipo I (10.3\%) y II (22.4\%), problemas tiroideos (1.22 veces más riesgo), en pacientes con enfermedad cerebrovascular con tratamiento quirúrgico para hemorragia subaracnoidea se reporta una prevalencia de $25.3 \%$ y enfermedad de Dupuytren asociada hasta en $50 \%$ de los casos. ${ }^{1,3,8}$

Diferentes estructuras morfológicas se ven afectadas como la bursa subacromial, la cápsula en la que hay pérdida de la capa sinovial, contractura capsular, adherencias en el tejido del receso axilar entre el mismo tejido y/o el cuello anatómico con disminución del volumen capsular que va de un promedio de $0.88 \mathrm{a} \mathrm{ml} \mathrm{a} 0.53$ en la capsulitis adhesiva, fibrosis del intervalo rotador, engrosamiento y contractura del ligamento coracohumeral de valores aproximados de 2.7 in the literature are reliable for the diagnosis of adhesive capsulitis. The low concordance with the imaging cabinets leads us to think that it is an underdiagnosed entity.

Keywords: Diagnostic, magnetic resonance, capsulitis, shoulder, surgery.

mm en el hombro sano a $4.1 \mathrm{~mm}$ y de la porción larga del bíceps al encontrarse contenida en el intervalo rotador. ${ }^{1}$

En la región superior y anterior de la cápsula se han observado distintos marcadores como los inmunológicos que incluyen al (TNF- $\alpha$ ), IL $1 \alpha$ y $\beta$ e IL-6, MMP-3, proteína quinasas activadas por mitógeno (ERK y JNK), NF- $\kappa$ B, TGF- $\beta 1$, CD29 (integrina b-1) y VEGF, marcadores para vasos sanguíneos (CD34), proteínas neuronales inmunorreactivas como la proteína asociada al crecimiento 43 (GAP43), el producto del gen de la proteína 9.5 (PGP9.5) y receptor del factor de crecimiento nervioso (P75) asociadas a regeneración de fibras nerviosas causantes del dolor, la vimentina una proteína contráctil encontrada en ensayos de inmunocitoquímica asociada a fibroblastos, que según Uhthoff y Boileau por medio de la actina se diferencian de miofibroblastos detectados en otras enfermedades como Dupuytren y otras fibromatosis. Se observa la presencia de células inflamatorias como factor de necrosis tumoral, linfocitos B, mastocitos y macrófagos y aumento de la vascularidad. En el estroma de la cápsula articular y en la bursa se ha notado aumento en la expresión de COX2 y en las células endoteliales incremento en la producción de COX1. ${ }^{4}$ Se cree que la capsulitis adhesiva es el resultado de una pérdida del equilibrio entre la degeneración,

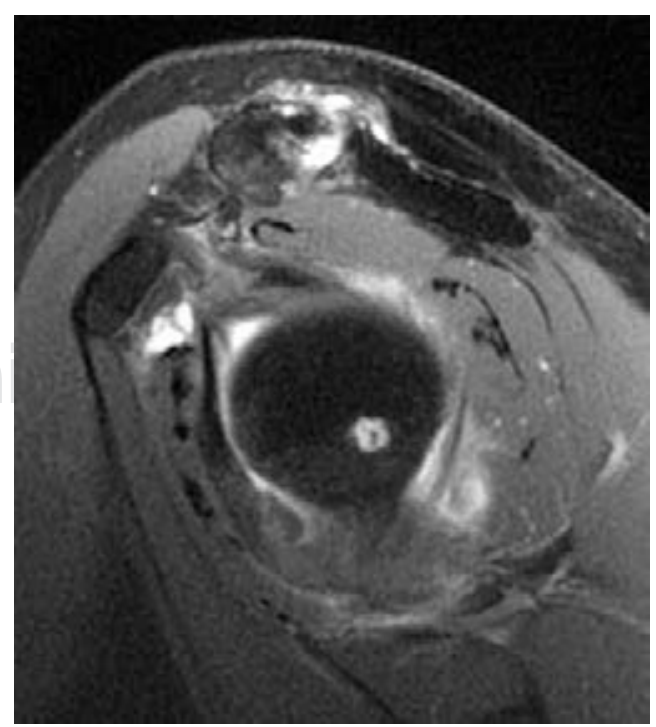

Figura 1: Engrosamiento del ligamento coracohumeral. 


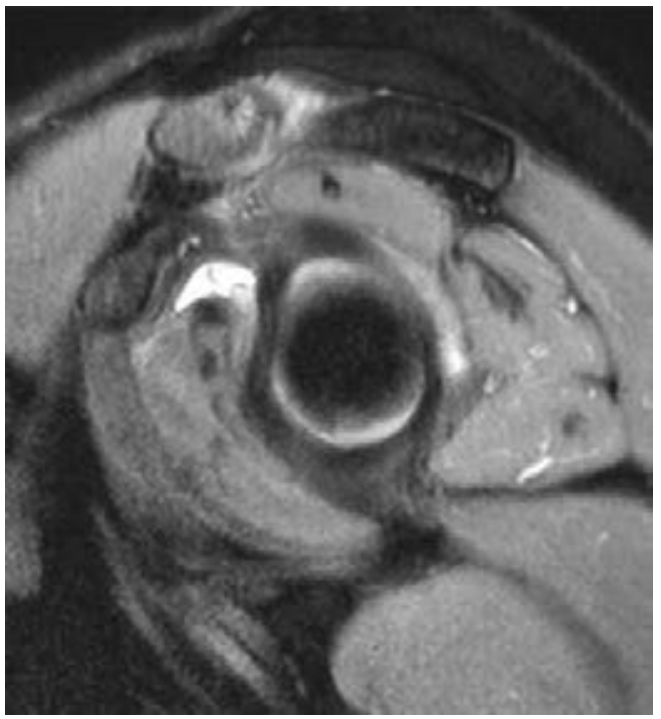

Figura 2: Infiltración del intervalo rotador y obliteración parcial de grasa subcoracoidea.

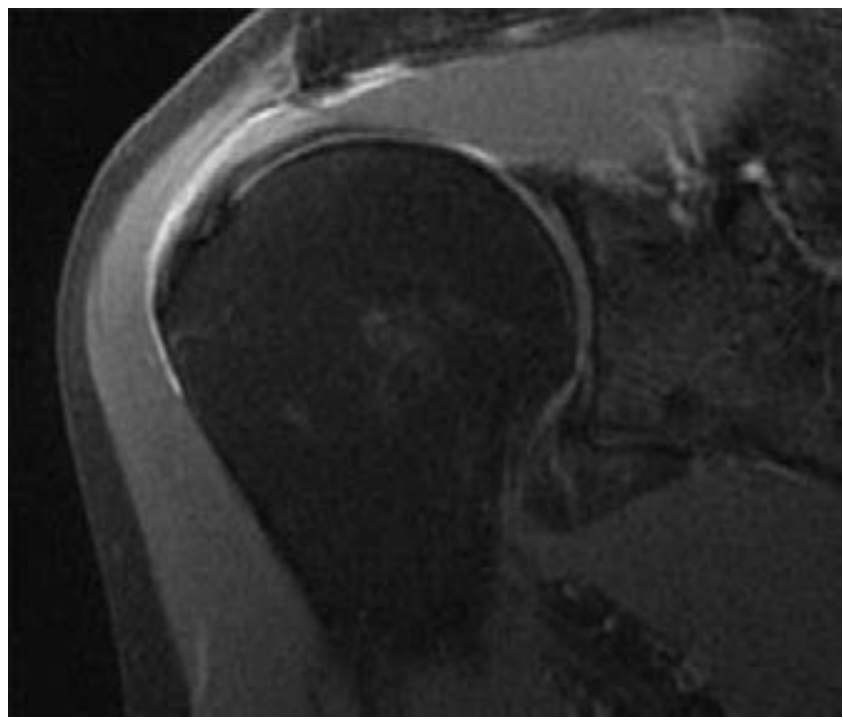

Figura 3: Engrosamiento capsular y contractura del receso axilar.

remodelación y regeneración en el tejido de matriz extracelular. ${ }^{1}$

Los datos radiológicos de capsulitis adhesiva en resonancia magnética han sido ampliamente descritos en la literatura, ${ }^{9}$ algunos de los cuales tienen una sensibilidad registrada desde $59 \%$ hasta $91 \%$ y una especificidad desde $53 \%$ hasta $100 \%$ en algunos reportes. ${ }^{1,3,6}$ Los datos valorados con más frecuencia son el engrosamiento del ligamento coracohumeral $>4 \mathrm{~mm}$ (Figura 1), infiltración grasa del intervalo rotador (Figura 2), engrosamiento capsular del receso axilar con valores que varían en diferentes estudios pero que se describe en general como $>7 \mathrm{~mm}$ (Figura 3), hiperintensidad o engrosamiento del ligamento glenohumeral inferior $>$ $3 \mathrm{~mm}$, obliteración de la grasa subcoracoidea descrita como ausente, parcial o completa (Figuras 1 y 2) y la contractura del receso axilar (Figura 3).

El objetivo del presente estudio es determinar la concordancia entre la interpretación realizada por un cirujano experto en hombro de la clínica de reconstrucción articular y los reportes de interpretación de diferentes centros de imagenología y compararlos con los hallazgos quirúrgicos.

Como hipótesis del estudio, se considera que existe una tendencia entre los centros de imagenología a ignorar los signos de la presencia de capsulitis adhesiva en los reportes de los estudios de imagen enviados con esa impresión diagnóstica.

\section{Material y métodos}

Se realizó un estudio retrospectivo, observacional y comparativo en pacientes con diagnóstico clínico-imagenológico de capsulitis adhesiva para determinar la concordancia entre los reportes de resonancia magnética nuclear de distintos gabinetes de radiología y la interpretación de la clínica de reconstrucción articular de hombro y codo; posteriormente la correlación entre los hallazgos radiológicos contra los hallazgos quirúrgicos.

El período de estudio comprendió de 2008 a 2018, durante el cual se encontraron en total 47 casos de capsulitis adhesiva, se incluyeron pacientes en cualquier rango de edad, sin importar el tiempo de evolución, se excluyeron pacientes sin resonancia magnética, obteniendo el registro de 41 casos para análisis, en 34 de estos pacientes el diagnóstico fue establecido por la exploración clínica inicial, en los otros siete el diagnóstico fue establecido posteriormente mediante el análisis de la resonancia magnética. Del total, 30 (74\%) fueron mujeres, 11 (26\%) fueron hombres, con una edad media de $52.43 \pm 8.5$ con un rango entre 35 y 67 años, el lado dominante fue el derecho en 39 casos (92.85\%) y el izquierdo en dos (4.76\%), el lado afectado

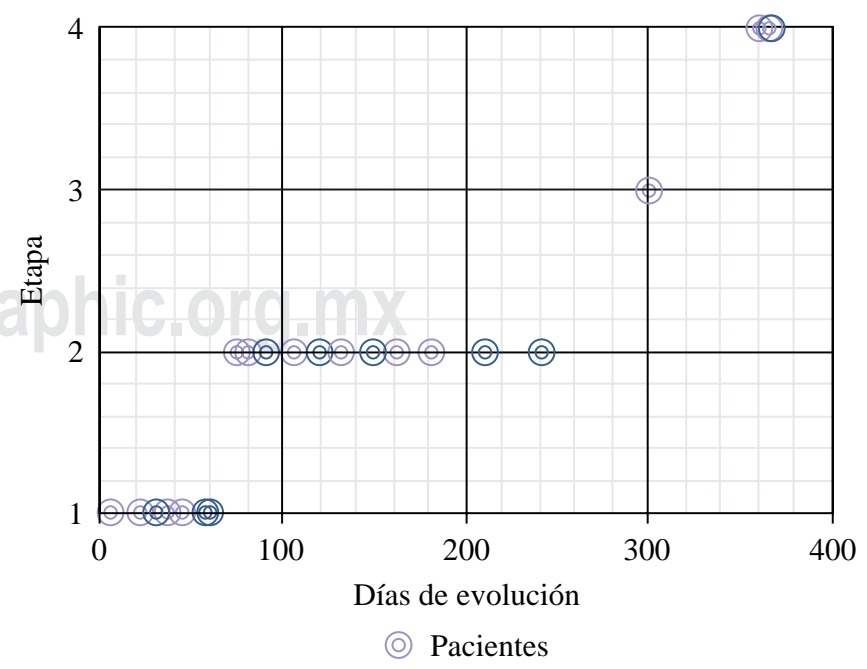

Figura 4: Distribución por etapas. 


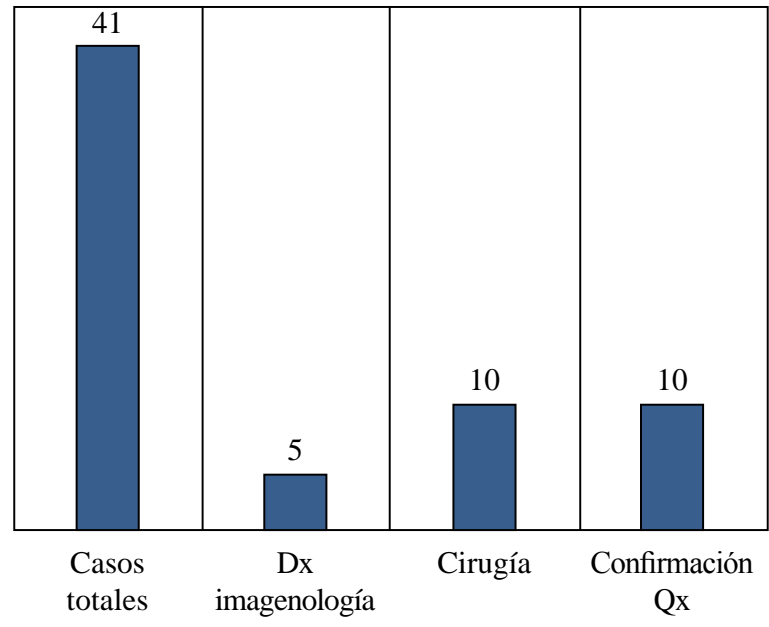

Figura 5: Casos diagnosticados por grupo y confirmación quirúrgica.

fue el derecho en 18 (45\%) y el izquierdo en 23 (55\%), el tiempo de evolución medio fue de $129.63 \pm 43.2$ días (Figura 4). Como patologías concomitantes se registraron tres casos de diabetes mellitus, siete casos de hipotiroidismo, cinco de hipertensión arterial sistémica, tres casos de artritis reumatoide, tres casos de disautonomía cardiaca, dos casos de asma, un caso de lupus, un caso de fibromialgia y un caso de CA de mama.

Se revisaron los diagnósticos clínicos iniciales, las interpretaciones de resonancia magnética realizadas por el cirujano con mayor experiencia en patología de hombro de la clínica de reconstrucción articular y se compararon con las interpretaciones de los centros de imagenología para obtener la concordancia entre ambas interpretaciones para el diagnóstico de capsulitis adhesiva. Los pacientes con diagnóstico inicial de capsulitis fueron enviados con la sospecha diagnóstica en la solicitud a los centros de imagenología, mientras que la interpretación de la RM por el cirujano de mayor experiencia se realizó previo a conocer el diagnóstico emitido por el médico radiólogo, en la imagen se buscaron datos característicos de capsulitis adhesiva como son: disminución del volumen articular, borramiento del receso axilar, engrosamiento capsular, engrosamiento del LGHI e infiltración del intervalo rotador. 31 pacientes fueron tratados con manejo conservador que constó de corticosteroides sistémicos e intraarticulares, AINES y terapia física y rehabilitación, mientras que 10 pacientes se trataron de manera quirúrgica con artroscopia, realizando artrofibrinólisis y sinovectomía más el tratamiento de las lesiones asociadas en los casos en los que se presentaron, comprobando en todos los casos la presencia de capsulitis adhesiva. Por último, se valoró la concordancia entre los hallazgos artroscópicos y las interpretaciones del experto y de los centros de imagen de los pacientes quirúrgicos para el diagnóstico de capsulitis adhesiva. Para el análisis estadístico se utilizaron medidas de tendencia central y pruebas de normalidad; para la comparación entre grupos t de Student, comparar frecuencias $\chi^{2}$ y correlaciones se utilizó un método de correlación lineal y para la concordancia se utilizó una prueba $\kappa$ en el programa estadístico SPSS versión 11 para Windows.

\section{Resultados}

De los reportes analizados de resonancia magnética el diagnóstico comparativo entre el experto y los reportes radiológicos de los gabinetes coincidieron en el diagnóstico de capsulitis adhesiva en cinco de los 41 casos (Figura 5), se observó baja concordancia $\kappa$ de 0.12 a pesar de contar con datos clínicos enviados por el servicio tratante. Los hallazgos radiográficos descritos por los diferentes grupos, adicionales a los datos de capsulitis adhesiva se muestran en las Tablas 1 y 2. En los pacientes que se trataron de forma quirúrgica por el experto y su equipo mediante artroscopia se encontró la presencia de artrofibrosis en los 10 casos, sólo uno de los 10 pacientes contaba con reporte de resonancia magnética con diagnóstico previo de capsulitis por parte de los gabinetes, estableciendo una baja correlación $\kappa$ de 0.10 , el resto de los hallazgos quirúrgicos se describen en la Tabla 3.

\section{Tabla 1: Lesiones reportadas en resonancia} magnética por gabinetes.

Tendinitis del supraespinoso

Tendinitis del infraespinoso

Desgarro redondo menor

Tenosinovitis subescapular

Tendinitis de porción larga del bíceps

Rotura del supraespinoso

Rotura del infraespinoso

Rotura del redondo menor

Rotura subescapular

Rotura del bíceps

Artrosis acromioclavicular

Artrosis glenohumeral

Desgarro de labrum

Quistes subperiósticos de la cabeza humeral

Lesión del ligamento glenohumeral medio

SLAP

Bursitis subescapular

Bursitis subcoracoidea

Bursitis subacromial

Infiltración grasa deltoides

Infiltración grasa infraespinoso

Infiltración grasa supraespinoso

Quistes en troquín o troquíter

Quistes en cabeza humeral

Edema óseo cabeza

Hipotrofia del deltoides

Hipotrofia del supraespinoso

Hipotrofia del infraespinoso

Hipotrofia del redondo menor

Capsulitis adhesiva

Engrosamiento de la cápsula

Pinzamiento subacromial

Fractura de tuberosidad mayor

Hipertrofia sinovial

Lipoma que comprime el trayecto del supraespinoso

Sinovitis acromioclavicular 
Tabla 2: Lesiones reportadas en resonancia magnética por el equipo de cirugía de hombro y codo.

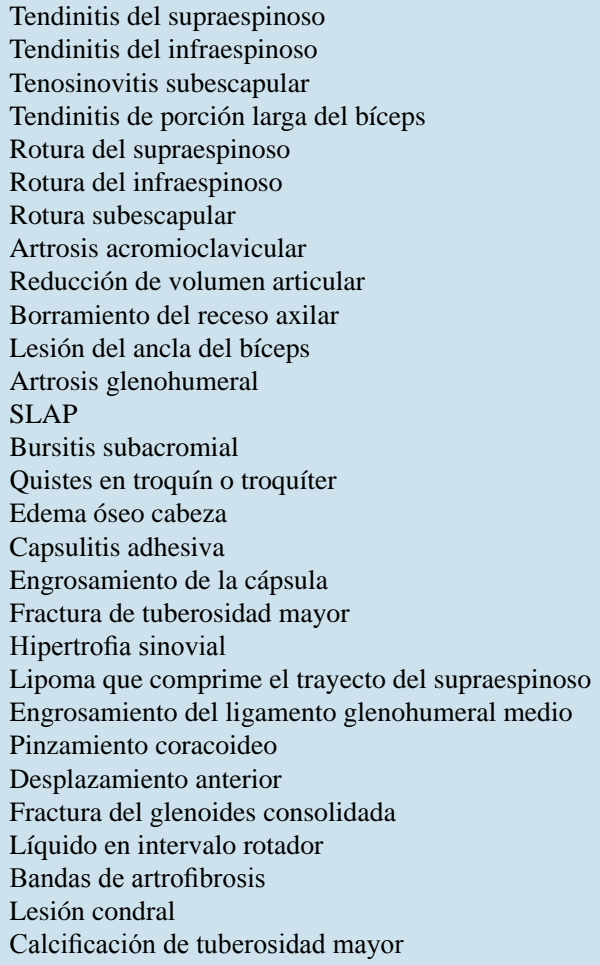

\section{Discusión}

Clínicamente la capsulitis adhesiva se presenta como un hombro doloroso que se exacerba por la noche y con limitación progresiva de los arcos de movimiento pasivos y activos. ${ }^{3,4}$ La rotación externa es el arco de movimiento más afectado, se puede definir como una pérdida mayor de $50 \%$, una rotación externa menor de $30^{\circ}$ o una pérdida de $25 \%$ en dos arcos o más de movimiento. ${ }^{10}$ En nuestra serie en la rotación externa media fue de $15^{\circ}$ en la etapa $1,10^{\circ}$ en el grupo clasificado en la etapa $2,30^{\circ}$ en la etapa 3 y $30^{\circ}$ en la etapa 4; para la flexión la mediana fue de $75^{\circ}, 80^{\circ}, 60^{\circ}$ y $80^{\circ}$, mientras que la abducción fue de $47.5^{\circ}, 60^{\circ}, 40^{\circ}$ y $70^{\circ}$ para las etapas 1 a 4 respectivamente.

Neviaser dividió el curso clínico de la capsulitis en cuatro etapas según los hallazgos artroscópicos, mientras que Reeves la dividió en tres etapas según el tiempo de evolución y sintomatología, los tiempos de evolución son variables en la literatura. ${ }^{10}$ En la etapa 1 o preadhesiva, los pacientes se quejan de dolor en el hombro de predominio nocturno, en la artroscopía hay evidencia de sinovitis sin adherencias ni contracturas. En la etapa 2, también llamada proliferativa o de congelamiento, ${ }^{1}$ que se presenta entre la semana 10 y 36,,$^{3,11}$ los pacientes comienzan a desarrollar rigidez, en artroscopía se observa nuevamente sinovitis con pérdida del receso axilar, sugerente de formación temprana de adherencias y contractura capsular. El estadio 3 de maduración o rigidez que se manifiesta de los cuatro a los 12 meses, ${ }^{3}$ se caracteriza por una pérdida global profunda de arcos de movimiento y dolor en los extremos. Durante esta etapa la sinovitis se resuelve, pero el pliegue axilar es obliterado como resultado de importantes adherencias. La etapa 4, también llamada etapa crónica o de descongelamiento, se presenta entre los 12 y 42 meses, ${ }^{3}$ en ella persiste la rigidez y el dolor es mínimo a medida que la sinovitis se ha resuelto. ${ }^{1,6}$ Es en la etapa 1 donde histológicamente se observan células inflamatorias en la sinovial, en la etapa 2 existe una proliferación sinovial y en la etapa 3 se observa engrosamiento del tejido de colágeno dentro de la cápsula. ${ }^{1}$ En nuestro estudio considerando el tiempo de evolución 14 pacientes se encontraban en fase preadhesiva, 23 en fase de congelamiento en la que incluimos pacientes con evolución hasta los nueve meses (36 semanas), debido a la sobreposición en las etapas de Reeves entre los cuatro y nueve meses, de los 23 pacientes, siete estaban entre las 10 semanas y los cuatro meses, 16 entre los cuatro y nueve meses, un paciente en fase de maduración que consideramos entre los nueve y 12 meses para evitar la sobreposición con el estadio 2, consideramos tres en etapa de descongelamiento con más de un año de evolución. Cinco pacientes se llevaron a tratamiento quirúrgico en fase preadhesiva y cinco en fase de congelamiento, uno antes de los cuatro meses y cuatro entre los cuatro y los nueve meses de evolución (Figura 4). Los pacientes que se llevaron a tratamiento quirúrgico tenían lesiones concomitantes o no presentaban mejoría del dolor con tratamiento conservador a base de analgésicos, antiinflamatorios, corticosteroides de depósito y terapia física y rehabilitación. En todos los pacientes sometidos a cirugía fue necesario realizar artrofibrinólisis independientemente de la etapa que cursaban.

Como en muchas patologías, en la capsulitis adhesiva los hallazgos de imagen han surgido de la correlación con los hallazgos quirúrgicos, es frecuente encontrar lesiones concomitantes del manguito rotador, labrum y artropatías. Su valoración en pacientes con clínica de capsulitis es fiable para establecer el diagnóstico de capsulitis adhesiva. Chi y colaboradores revelaron que al usar los criterios de engrosamiento del ligamento coracohumeral, la infiltración del intervalo rotador y el engrosamiento del receso axilar la sensibilidad aumentaba de $53.3 \%$ de sólo la valoración del ligamento coracohumeral a $86.7 \%$ con el uso de los tres datos. ${ }^{9}$ La baja correlación ( $\kappa 0.12$ y 0.10 ) encontrada entre las

Tabla 3: Hallazgos artroscópicos.

Artrofibrosis

Sinovitis glenohumeral

Bursitis subacromial

Fibrosis del intervalo rotador

Tendinopatía del bíceps

Rotura del supraespinoso

Artrosis acromioclavicular Pinzamiento 


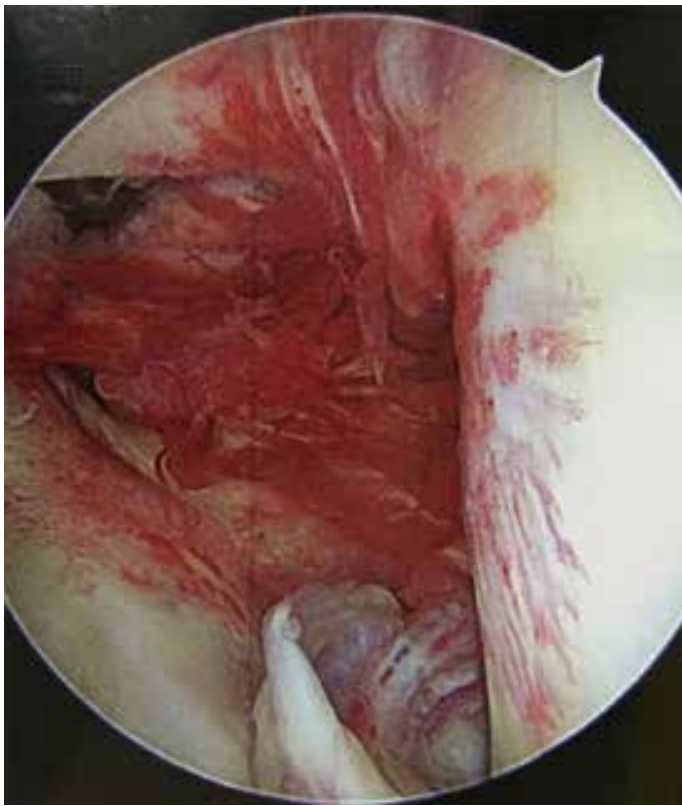

Figura 6: Adherencias en receso axilar y fibrosis del intervalo rotador que afecta la porción larga del bíceps, con sinovitis.

interpretaciones de los distintos gabinetes de radiología nos orienta a pensar que es una entidad subdiagnosticada a pesar de la sospecha clínica con la que se envían las solicitudes. $\mathrm{Al}$ ser una entidad de manejo conservador en la mayor parte de los casos es difícil establecer una correlación quirúrgica exacta con ambas interpretaciones.

Como se describe en la literatura se presentaron distintos factores asociados en nuestra serie, siendo el padecimiento más común el hipotiroidismo y algunas otras enfermedades relacionadas con padecimientos autoinmunes. En cuanto al género, también encontramos mayor asociación en pacientes femeninos.

El tratamiento se divide en tratamiento quirúrgico y no quirúrgico, el tratamiento conservador busca restaurar el movimiento, disminuir el dolor y la duración de los síntomas. ${ }^{1,3}$ Existe evidencia de que la terapia física mediante movilización temprana ofrece buenos resultados así como programas de estiramientos ${ }^{11}$ o fortalecimiento muscular. ${ }^{8}$ Según Diercks y Stevens la movilización gentil tuvo mejores resultados que la intensiva. ${ }^{1}$

Dentro de las opciones de tratamiento farmacológico contamos con los antiinflamatorios no esteroideos (AINES), corticosteroides sistémicos, intraarticulares o locales. Los corticosteroides se indican principalmente durante la fase inflamatoria temprana para alivio del dolor a corto plazo, se ha observado que mejoran la sintomatología nocturna cuando se administran de forma sistémica. El sitio de aplicación en la capsulitis adhesiva es controversial, existen estudios que demuestran que cuando se administran intraarticulares se nota mejoría en el curso clínico a corto plazo, ${ }^{1}$ mientras que a largo plazo se ha observado mejoría en rangos de movimiento, aunque en otros estudios no existe una diferencia estadísticamente significativa. ${ }^{11,12}$ También se reporta mejoría cuando éstos se aplican en el intervalo rotador. ${ }^{13}$

El ácido hialurónico por sus efectos en cartílago, líquido sinovial y sinovia contribuye a mejorar el curso clínico, algunos estudios han detectado mejora en los arcos de movimiento, la escala de Constant y el dolor. ${ }^{1}$ El bloqueo del nervio supraescapular con inervación sensitiva de $70 \%$ de la articulación glenohumeral, con resultados variables en dolor y movimiento a largo plazo., ${ }^{1,314}$ La hidrodilatación o artrografía de distensión con medio de contraste o solución salina y corticosteroides, con resultados discutibles en mejoría a corto plazo de dolor y movimiento. ${ }^{1,15}$ Existen otros tratamientos menos estudiados como crioterapia de cuerpo completo con terapia física, infiltración con toxina botulínica, ${ }^{1}$ embolización arterial ${ }^{16}$ o la terapia de ondas de choque con masaje miofascial en rango de 0.08 a $0.28 \mathrm{~mJ} / \mathrm{mm}^{2}$ y de 10 a $13 \mathrm{~Hz}$ en un rango de 400 a 500 ondas. ${ }^{17}$

Entre las opciones de tratamiento quirúrgico tenemos la manipulación bajo anestesia y la capsulectomía artroscópica o abierta. ${ }^{1,3}$ La liberación artroscópica reporta buenos resultados cuando se realiza la liberación de la porción larga del bíceps y del ligamento coracohumeral, ${ }^{18}$ cuando se libera sólo la región posteroinferior de la cápsula ${ }^{19} \mathrm{o}$ con la apertura del intervalo rotador con resección del ligamento coracohumeral. ${ }^{20}$ En nuestro estudio, los casos que se llevaron a tratamiento quirúrgico fueron los pacientes que no mostraron mejoría con el tratamiento conservador, sólo dos casos presentaron lesiones del supraespinoso y seis casos de tendinopatía del bíceps que requirió tenodesis, uno de los cuales tenía un lipoma. Se comprobó la presencia de sinovitis (Figuras 6 a 9), infiltración del intervalo rotador (Figuras 6 y 7), adherencias y obliteración del receso axilar (Figura 10), tendinopatía del bíceps (Figuras 6 y 9) y engrosamiento del ligamento glenohumeral medio (Figura 8). En todos los casos se realizó artrofibrólisis artroscópica,

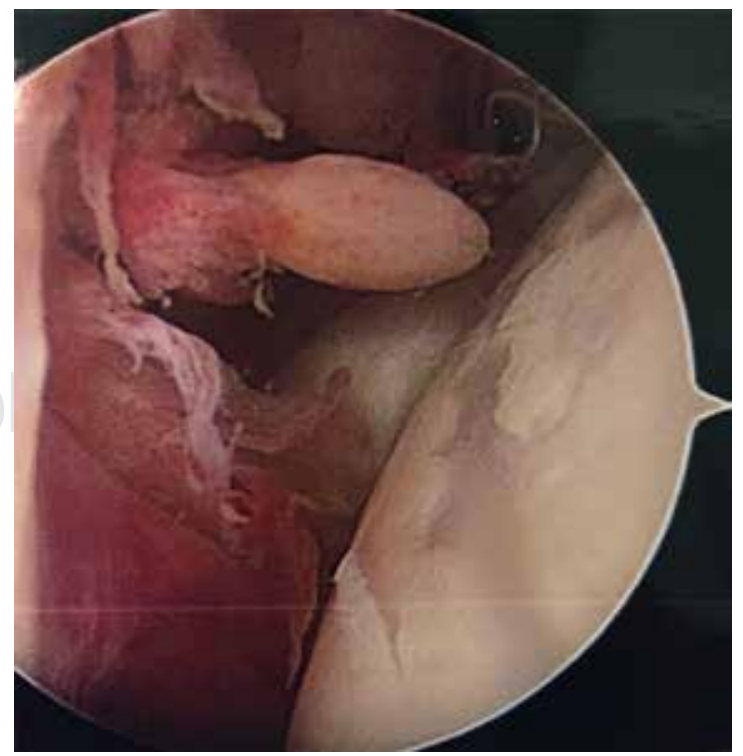

Figura 7: Fibrosis del intervalo rotador con sinovitis. 
sinovectomía, en dos aperturas del intervalo rotador (Figura 11). Las lesiones del supraespinoso y del lipoma se reportaron en ambas interpretaciones. Debido a la buena respuesta de los pacientes al tratamiento conservador en la mayor parte de los casos no resulta posible corroborar quirúrgicamente $100 \%$ de los casos tratados.

Una limitante de este estudio es que la descripción de los datos en ambos reportes se realizó de manera cualitativa y no se registraron las mediciones de los datos encontrados en las resonancias analizadas.

Otro aspecto que podría considerarse como una limitante es el hecho de que sólo se tuvo a un evaluador del área ortopédica con entrenamiento en patología de hombro, lo que podría distorsionar los resultados al suponer que todos los cirujanos ortopedistas tienen el conocimiento para establecer el diagnóstico de capsulitis adhesiva usando la resonancia magnética, por lo que consideramos que sería conve-

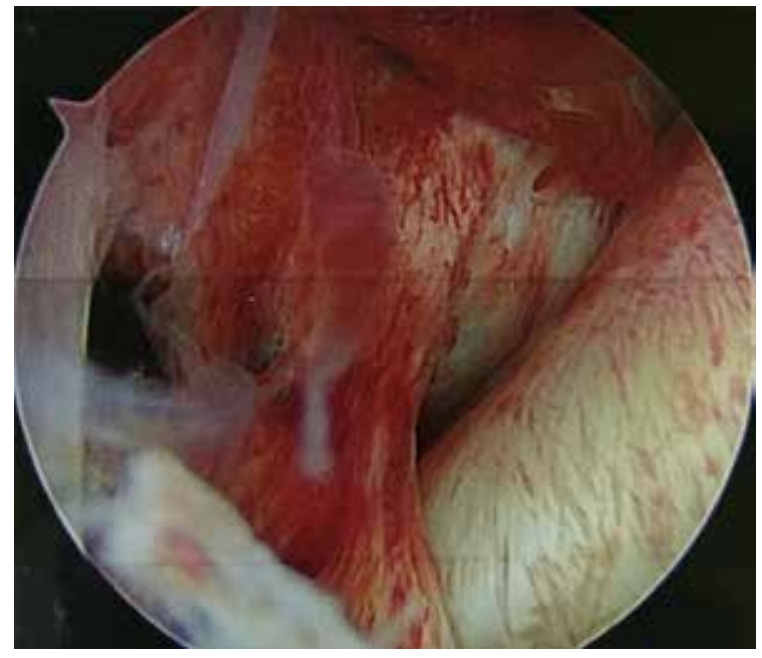

Figura 8: Engrosamiento del ligamento glenohumeral medio y sinovitis.

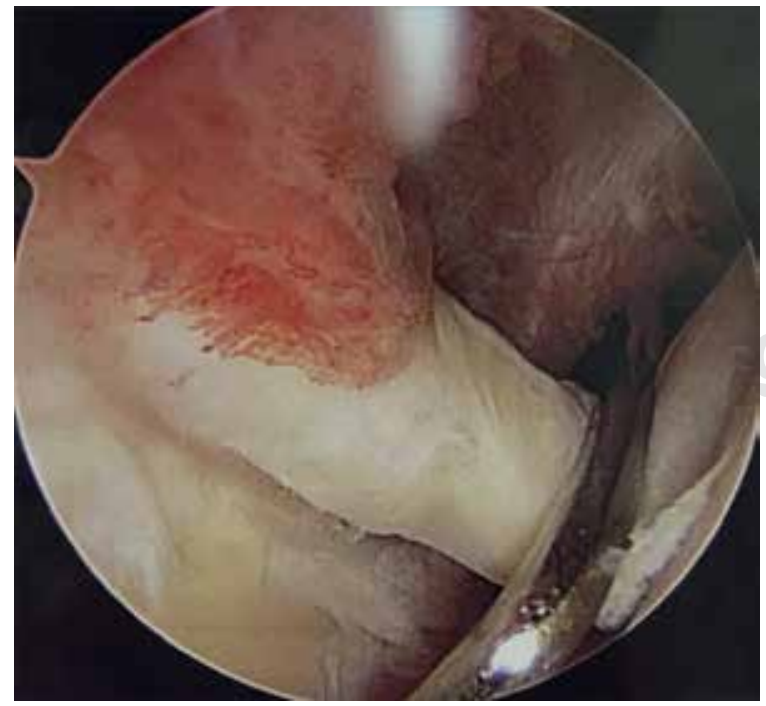

Figura 9: Tendinopatía del tendón de la porción larga del bíceps.

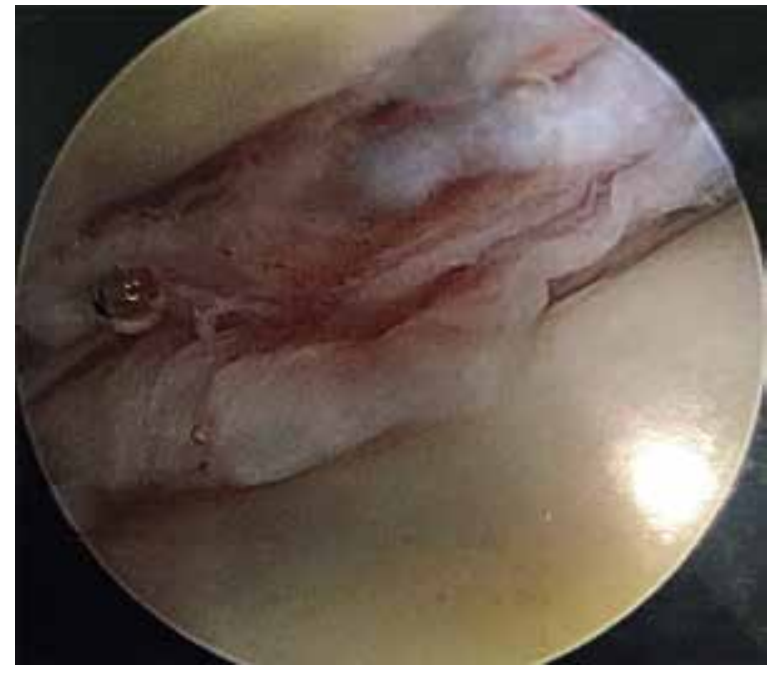

Figura 10: Adherencias en receso axilar.

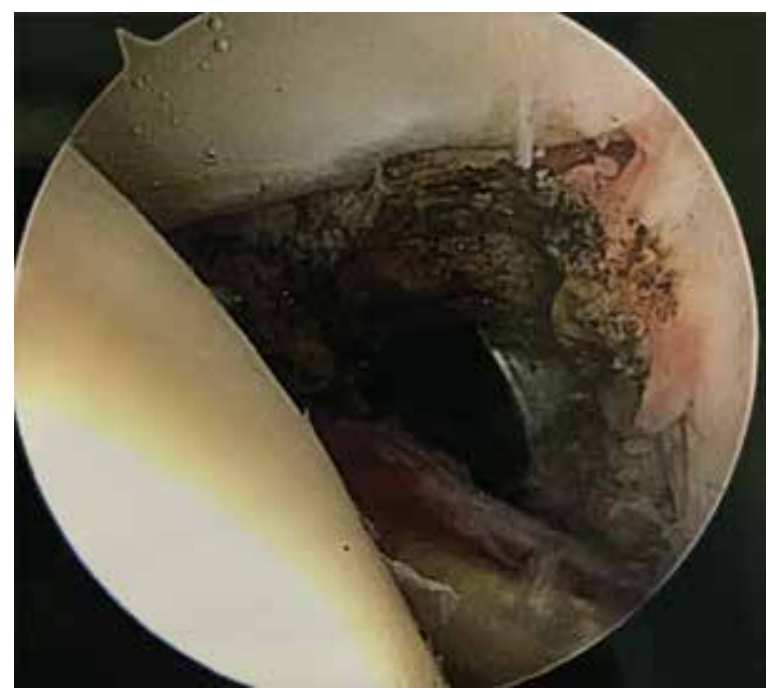

Figura 11: Apertura del intervalo rotador.

niente realizar un estudio de concordancia intraobservador e interobservador con diferentes evaluadores de diferentes grados de entrenamiento en ambas áreas: ortopedia e imagenología, para determinar el grado de afectación de esta variable en el diagnóstico oportuno de esta afección.

\section{Conclusiones}

Se corroboró la hipótesis de trabajo, con base en los resultados obtenidos de la falta de concordancia entre los centros de imagenología y la clínica de reconstrucción articular. Los datos radiológicos descritos en resonancia magnética para el diagnóstico de capsulitis son confiables, de acuerdo a lo demostrado en los pacientes en quienes se llevó a cabo el procedimiento quirúrgico, 100\% correspondió al diagnóstico establecido tras la interpretación de la resonancia magnética. Los médicos tratantes tenemos la oportunidad 
de orientar nuestro diagnóstico por la clínica y con base en esto buscar datos específicos en resonancia que corroboren nuestra sospecha. Sin embargo, las solicitudes que son enviadas a los diferentes gabinetes establecen un diagnóstico presuntivo, que resulta ignorado en los reportes finales de los centros de imagen, lo que ocasiona un subdiagnóstico de la enfermedad.

Bibliografía

1. Le H V, Lee SJ, Nazarian A, Rodriguez EK. Adhesive capsulitis of the shoulder: review of pathophysiology and current clinical treatments. Shoulder Elb. 2017; 9(2): 75-84. doi: 10.1177/1758573216676786.

2. Candela V, Giannicola G, Passaretti D, Venditto T, Gumina S. Adhesive capsulitis of the shoulder: pain intensity and distribution. Musculoskelet Surg. 2017; 101(s2): 153-8. doi:10.1007/s12306-0170488-6.

3. Zappia M, Di Pietto F, Aliprandi A, et al. Multi-modal imaging of adhesive capsulitis of the shoulder. Insights Imaging. 2016; 7(3): 36571. doi:10.1007/s13244-016-0491-8.

4. Ryan V, Brown H, Minns Lowe CJ, Lewis JS. The pathophysiology associated with primary (idiopathic) frozen shoulder: A systematic review. BMC Musculoskelet Disord. 2016; 17(1): 340. doi: 10.1186/ s12891-016-1190-9.

5. Lamplot JD, Lillegraven O, Brophy RH. Outcomes from conservative treatment of shoulder idiopathic adhesive capsulitis and factors associated with developing contralateral disease. Orthop J Sport Med. 2018; 6(7): 232596711878516. doi:10.1177/2325967118785169.

6. Park GY, Park JH, Kwon DR, Kwon DG, Park J. Do the findings of magnetic resonance imaging, arthrography, and ultrasonography reflect clinical impairment in patients with idiopathic adhesive capsulitis of the shoulder? Arch Phys Med Rehabil. 2017; 98(10): 1995-2001. doi:10.1016/j.apmr.2017.06.006.

7. Lee M, Theodoulou A, Krishnan J. Criteria used for diagnosis of adhesive capsulitis of the shoulder. JBI Database Syst Rev Implement Reports. 2018; 16(6): 1332-7. doi:10.11124/JBISRIR-2017-003609.

8. Rawat P, Eapen C, Seema KP. Effect of rotator cuff strengthening as an adjunct to standard care in subjects with adhesive capsulitis: a randomized controlled trial. $J$ Hand Ther. 2017; 30(3): 235-41.e8. doi:10.1016/j.jht.2016.10.007.
9. Chi AS, Kim J, Long SS, Morrison WB, Zoga AC. Non-contrast MRI diagnosis of adhesive capsulitis of the shoulder. Clin Imaging. 2017; 44: 46-50. doi: 10.1016/j.clinimag.2017.04.002

10. Kelley MJ, Shaffer MA, Kuhn JE, et al. Shoulder pain and mobility deficits: adhesive capsulitis. J Orthop Sport Phys Ther. 2013; 43(5): A1-31. doi:10.2519/jospt.2013.0302

11. Yip M, Francis A-M, Roberts T, Rokito A, Zuckerman JD, Virk MS Treatment of adhesive capsulitis of the shoulder a critical analysis review. JBJS Rev. 2018; 6(6): e5. doi: 10.2106/JBJS.RVW.17.00165.

12. Wang W, Shi M, Zhou C, et al. Effectiveness of corticosteroid injections in adhesive capsulitis of shoulder. Medicine (Baltimore). 2017; 96(28): e7529.

13. Sun Y, Liu S, Chen S, Chen J. The effect of corticosteroid injection into rotator interval for early frozen shoulder: a randomized controlled trial. Am J Sports Med. 2018; 46(3): 663-70. doi: $10.1177 / 0363546517744171$.

14. Prasanna KJ, Rajeswari R, Sivakuma VPR. Effectiveness of scapular proprioceptive neuromuscular facilitation (PNF) techniques in adhesive capsulitis of the shoulder joint. J Physiother Res. 2017; 1(2): 9.

15. Saltychev M, Laimi K, Virolainen P, Fredericson M. Effectiveness of hydrodilatation in adhesive capsulitis of shoulder: a systematic review and meta-analysis. Scand J Surg. 2018; 1457496918772367. doi: $10.1177 / 1457496918772367$.

16. Okuno Y, Iwamoto W, Matsumura N, et al. Clinical outcomes of transcatheter arterial embolization for adhesive capsulitis resistant to conservative treatment. J Vasc Interv Radiol. 2017; 28(2): 161-7.e1. doi: 10.1016/j.jvir.2016.09.028

17. Yuan X, Zhou F, Zhang L, Zhang Z, Li J. Analgesic effect of extracorporeal shock wave treatment combined with fascial manipulation theory for adhesive capsulitis of the shoulder: a retrospective study. Biomed Res Int. 2018; 2018: 3450940.

18. Kanbe K. Clinical outcome of arthroscopic capsular release for frozen shoulder: Essential technical points in 255 patients. J Orthop Surg Res. 2018; 13(1): 1-7. doi:10.1186/s13018-018-0758-5.

19. Puah KL, Salieh MS, Yeo W, Tan AHC. Outcomes of arthroscopic capsular release for the diabetic frozen shoulder in Asian patients. J Orthop Surg. 2018; 26(1): 230949901875757. doi: 10.1177/2309499018757572.

20. Miyazaki AN, Santos PD, Silva LA, Sella G do V, Carrenho L, Checchia SL. Clinical evaluation of arthroscopic treatment of shoulder adhesive capsulitis. Rev Bras Ortop (English Ed). 2017; 52(1): 61-8. doi: 10.1016/j.rboe.2016.12.004. 